\title{
Systemic Lupus Erythematosus Associated with Ovarian Cancer
}

\author{
Mayu Yagita ${ }^{1}$, Saori Hata $^{2}$, Hiromi Miyata ${ }^{2}$, Hiroko Kakita ${ }^{3}$, Tatsuo Tsukamoto ${ }^{3}$, \\ Eri Muso $^{3}$ and Masaaki Fujita ${ }^{1}$
}

\begin{abstract}
:
Systemic lupus erythematosus (SLE) may be associated with various types of malignancy. However, SLE occurring with ovarian cancer seems rare, and reliable therapeutic approaches for such cases have yet to be identified. We herein report a case of SLE with ovarian cancer that was successfully treated with corticosteroid, plasmapheresis and chemotherapy. This case may provide new insights into treatment approaches for SLE with ovarian cancer.
\end{abstract}

Key words: systemic lupus erythematosus, ovarian cancer, malignancy

(Intern Med 58: 731-735, 2019)

(DOI: 10.2169/internalmedicine.1736-18)

\section{Introduction}

Systemic lupus erythematosus (SLE) is a systemic chronic inflammatory disease. Corticosteroids and immunosuppressants are the key drugs used to treat SLE (1). Patients with SLE have a slightly higher overall risk of malignancy than those without SLE (2-8). Indeed, a recent study showed increased risks of non-Hodgkin's lymphoma, lung, liver, vaginal and thyroid malignancies but decreased risks of ovarian, breast and prostate cancer. Ovarian cancer with SLE appears to be extremely rare (0.1\% of SLE patients) (2). The decreased rates of these hormone-sensitive cancers are speculated to be related to the presence of lupus autoantibodies and the down-regulation of certain pathways in $\operatorname{SLE}(7,8)$.

We herein report a case of SLE with ovarian cancer that was successfully treated with corticosteroid, plasmapheresis and chemotherapy.

\section{Case Report}

A 49-year-old woman was diagnosed with ovarian cancer at another hospital (Fig. 1). She was referred to the gynecology department at our hospital, and chemotherapy had been planned. However, a physical examination showed arthritis, oral ulcer and pitting edema of the distal extremities.

Laboratory testing showed the following: white blood cell count, $8,200 / \mu \mathrm{L}$; hemoglobin, $7.1 \mathrm{~g} / \mathrm{dL}$; platelets, $8.6 \times 10^{4} /$ $\mu \mathrm{L}$; albumin, $3.0 \mathrm{~g} / \mathrm{dL}$ (normal $>4.1 \mathrm{~g} / \mathrm{dL}$ ); urea nitrogen, $52.7 \mathrm{mg} / \mathrm{dL}$; creatinine, $3.7 \mathrm{mg} / \mathrm{dL}$; urine protein, $3,655 \mathrm{mg} /$ g Cre; anti-nuclear antibody, 1:80 (normal $<1: 40$ ); antidsDNA antibody, $17 \mathrm{IU} / \mathrm{mL}$ (normal $<12 \mathrm{IU} / \mathrm{mL}$ ); plateletassociated $\mathrm{IgG}, 117 \mathrm{ng} / 10^{7}$ cells (normal $<46 \mathrm{ng} / 10^{7}$ cells); C1q, $17.3 \mu \mathrm{g} / \mathrm{mL}$ (normal $<3 \mu \mathrm{g} / \mathrm{dL}$ ); C3, $67 \mathrm{mg} / \mathrm{dL}$ (normal 65-135 mg/dL); C4, $11 \mathrm{mg} / \mathrm{dL}$ (normal 13-35 mg/dL); CH50, $28 \mathrm{U} / \mathrm{mL}$ (normal $30-50 \mathrm{U} / \mathrm{mL}$ ) and a positive result for the direct Coombs test. Chest X-ray showed no abnormal findings in the lungs. Abdominal computed tomography showed no abnormalities in the liver, gallbladder, liver, or pancreas. A kidney biopsy revealed diffuse mesangial proliferation with segmental necrotizing crescent formation and vasculitis. Positive mesangial staining for IgG, IgA, IgM, C3 and $\mathrm{Clq}$ indicated class III lupus nephritis associated with vasculitis (Fig. 2). The patient met both the Systemic Lupus International Collaborating Clinics classification criteria and the revised criteria of the American College of Rheumatology (3-5). She was therefore diagnosed with SLE associated with ovarian cancer. The systemic lupus erythematosus dis-

${ }^{1}$ Department of Clinical Immunology and Rheumatology, The Tazuke-Kofukai Medical Research Institute, Kitano Hospital, Japan, ${ }^{2}$ Department of Obstetrics and Gynecology, The Tazuke-Kofukai Medical Research Institute, Kitano Hospital, Japan and ${ }^{3}$ Department of Nephrology and Dialysis, The Tazuke-Kofukai Medical Research Institute, Kitano Hospital, Japan

Received: June 26, 2018; Accepted: July 29, 2018; Advance Publication by J-STAGE: October 17, 2018

Correspondence to Dr. Masaaki Fujita, ms-fujita@kitano-hp.or.jp 


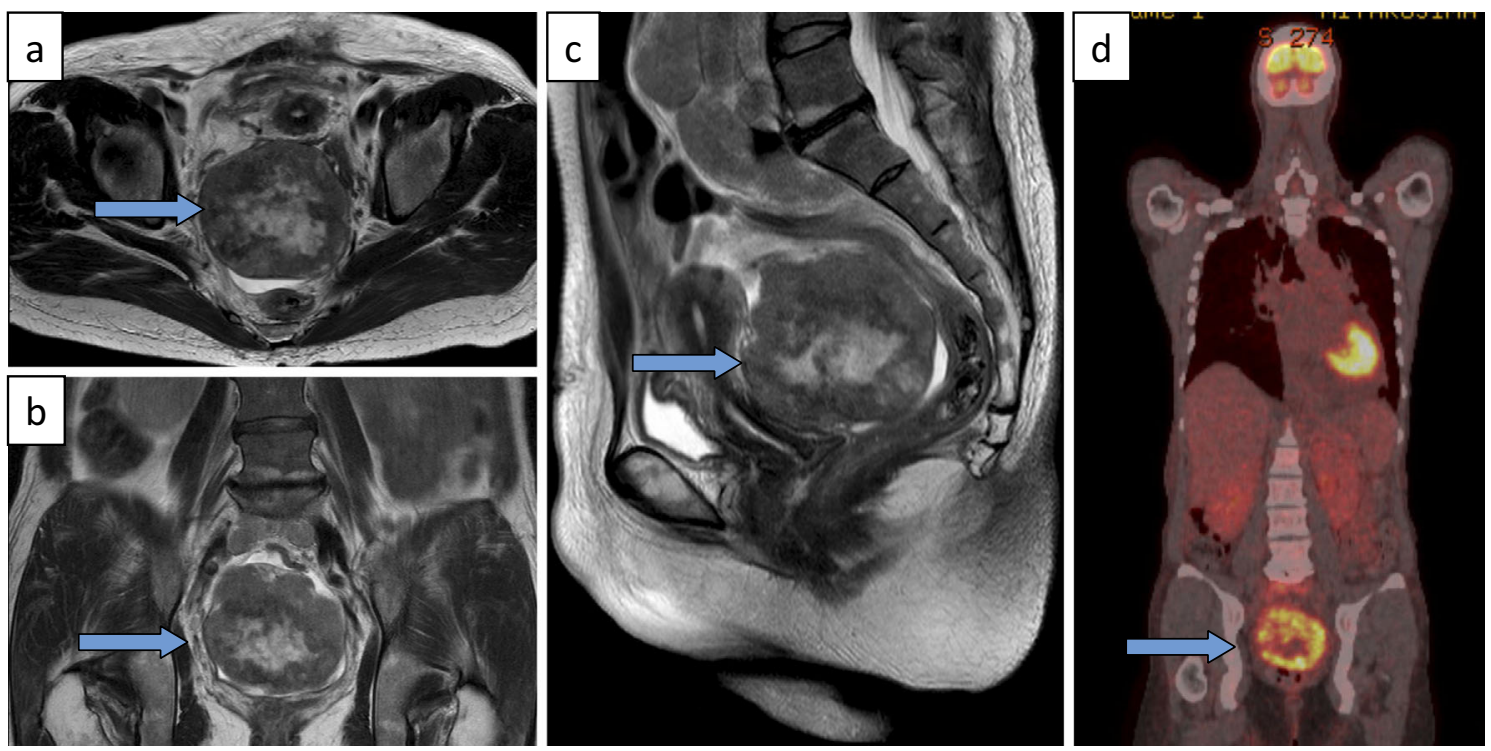

Figure 1. Images of the ovarian tumor. (a-c) T2-weighted MRI reveals the ovarian tumor $(72 \times 77 \times 64$ $\mathrm{mm}$ ). (d) PET-CT reveals the increased FDG uptake in the tumor. PET: positron emission tomography, FDG: fluorodeoxy glucose

ease activity index (SLEDAI) score was 29 the day before starting treatment (day 0$)$.

Oral prednisolone (1 mg/kg; $45 \mathrm{mg}$ ) was started (Fig. 3). In addition, she received intravenous methylprednisolone (1 $\mathrm{g} /$ day) for 3 days, and plasma exchange was performed as she developed rapidly progressive glomerulonephritis. We followed the general protocol used for SLE with rapidly progressive glomerulonephritis in Japan. Albumin was used as the replacement solution for the first plasma exchange, and fresh-frozen plasma was used for the second, third and fourth times. The estimated plasma volume (in liters) was calculated as follows: body weight $(\mathrm{kg}) \times(1$-hematocrit $) / 12$.

Her oral ulcer and arthritis rapidly resolved. Prednisolone was therefore tapered, and dose-dense paclitaxel and carboplatin chemotherapy (dose-dense TC) was initiated (carboplatin: Day 1 and paclitaxel: Day 1, 8, 15). The chemotherapy was repeated every three weeks for three cycles. The levels of serum creatinine and immune complexes and urine protein gradually decreased (day 80 : Cre, $0.84 \mathrm{mg} / \mathrm{dL}$; C1q, $3.6 \mu \mathrm{g} / \mathrm{mL}$; urine protein, $1,152 \mathrm{mg} / \mathrm{g}$ Cre). The SLEDAI score improved (day 30, 14; day 60, 12; day 210, 8). The ovarian tumor also decreased in size after three cycles of chemotherapy.

On day 90, she underwent total hysterectomy and bilateral salpingo-oophorectomy with pelvic and paraaortic lymph node dissection. The tumor size was $9 \times 6 \times 5 \mathrm{~cm}(148$ g). The tumor cells showed nuclear pleomorphism and tubular proliferation. Immunohistochemically, the tumor cells were positive for p53 (diffuse) and cytokeratin 7 and negative for cyclin-dependent kinase 2 and estrogen receptor (Fig. 4). The results of a pathological examination were thus compatible with ovarian high-grade serous adenocarcinoma with regional lymph node metastasis (FIGO Stage IVb).

After surgery, she received adjuvant chemotherapy with docetaxel and carboplatin (DC). The chemotherapy was repeated every three weeks for six cycles. The concentrations of serum creatinine and immune complexes remained low (day 210: Cre, $0.87 \mathrm{mg} / \mathrm{dL}$; C1q, $2.2 \mu \mathrm{g} / \mathrm{mL}$ ), and urine protein decreased even further (day 210: urine protein, $437 \mathrm{mg}$ / g Cre).

\section{Systematic review}

We conducted a systematic review of the Medline database through the PubMed website of articles published from 1966 to 2016 using the search terms "lupus" and "ovarian". Non-English and duplicate articles were excluded. We identified a total of two articles describing SLE with ovarian cancer reported as English full-text articles.

\section{Discussion}

Ovarian cancer with SLE is extremely rare. A metaanalysis revealed 53 cases of ovarian cancer among 54,573 patients with SLE (2). Furthermore, the risk of ovarian cancer is estimated to be relatively low (pooled relative risk, $0.76 ; 95 \%$ confidence interval, 0.56-1.01). The reason for the low rates of ovarian cancer among SLE patients is unclear. However, SLE patients have a decreased risk of several hormone-sensitive cancers (including ovarian, breast and endometrial cancer), suggesting the possible influence of an altered metabolism of estrogen and/or other hormones. Alternatively, some lupus autoantibodies may be related to the low rates of ovarian cancers observed among SLE patients.

Most self-reactive antibodies are critical for the pathogenesis of SLE. However, Hansen et al. showed the therapeutic potential of cell-penetrating lupus autoantibodies for breast cancer susceptibility gene II (BRCA2)-deficient human cancers (ovarian, pancreatic and colon can- 


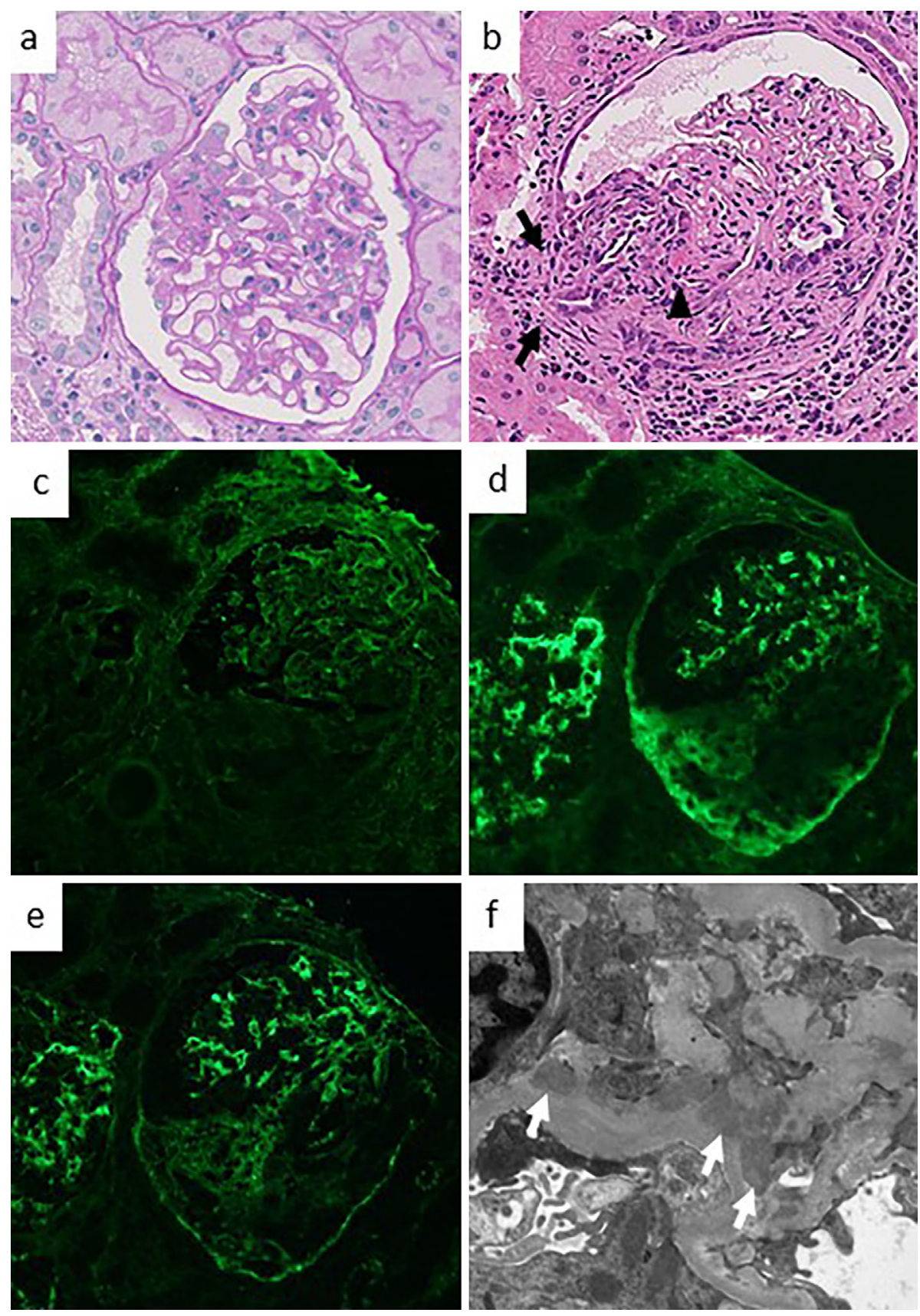

Figure 2. Renal histopathological findings. Microscopic findings: (a) Mesangial proliferation with necrosis (Periodic acid-schiff stain). (b) Tuft necrosis (arrowhead) with fibro-cellular crescent and arteriolitis (arrows) with inflammatory cell infiltration at the vascular pole. It is surrounded by active interstitial inflammation (Hematoxylin and Eosin staining). Positive immunofluorescence staining for IgG (c), C1q (d), C3c (e). Apparent mesangial electron-dense deposits (white arrows) as shown on electron microscopy $(\mathrm{f}: \times \mathbf{8 , 0 0 0})$.

cers) $(12,13)$. They suggest that lupus autoantibodies may be related to the low rates of BRCA2-associated malignancies, such as breast and ovarian cancers, among SLE patients (12). Therefore, a lack of these antibodies may have been related to the onset of ovarian cancer in our case. BRCA2 mutations were not investigated in the present case, although their existence would have been interesting to know. Of note, the patient had complained of metrorrhagia and been diagnosed with uterine myoma $(6 \times 7 \times 7 \mathrm{~cm})$ at another hospital 1 year before admission to our hospital. Ret- rospectively, that pathology is thought to have probably been ovarian cancer, as no uterine myoma was detected in tissue specimens after surgery. Although ovarian serous adenocarcinoma is an aggressive cancer, no marked changes in tumor size were seen for one year in our case. Therefore, lupus autoantibodies may have inhibited tumor progression before the onset of ovarian cancer.

To analyze the published evidence, we conducted a systematic review that identified two cases of SLE with ovarian cancer. In one case, a 34-year-old woman was diagnosed 


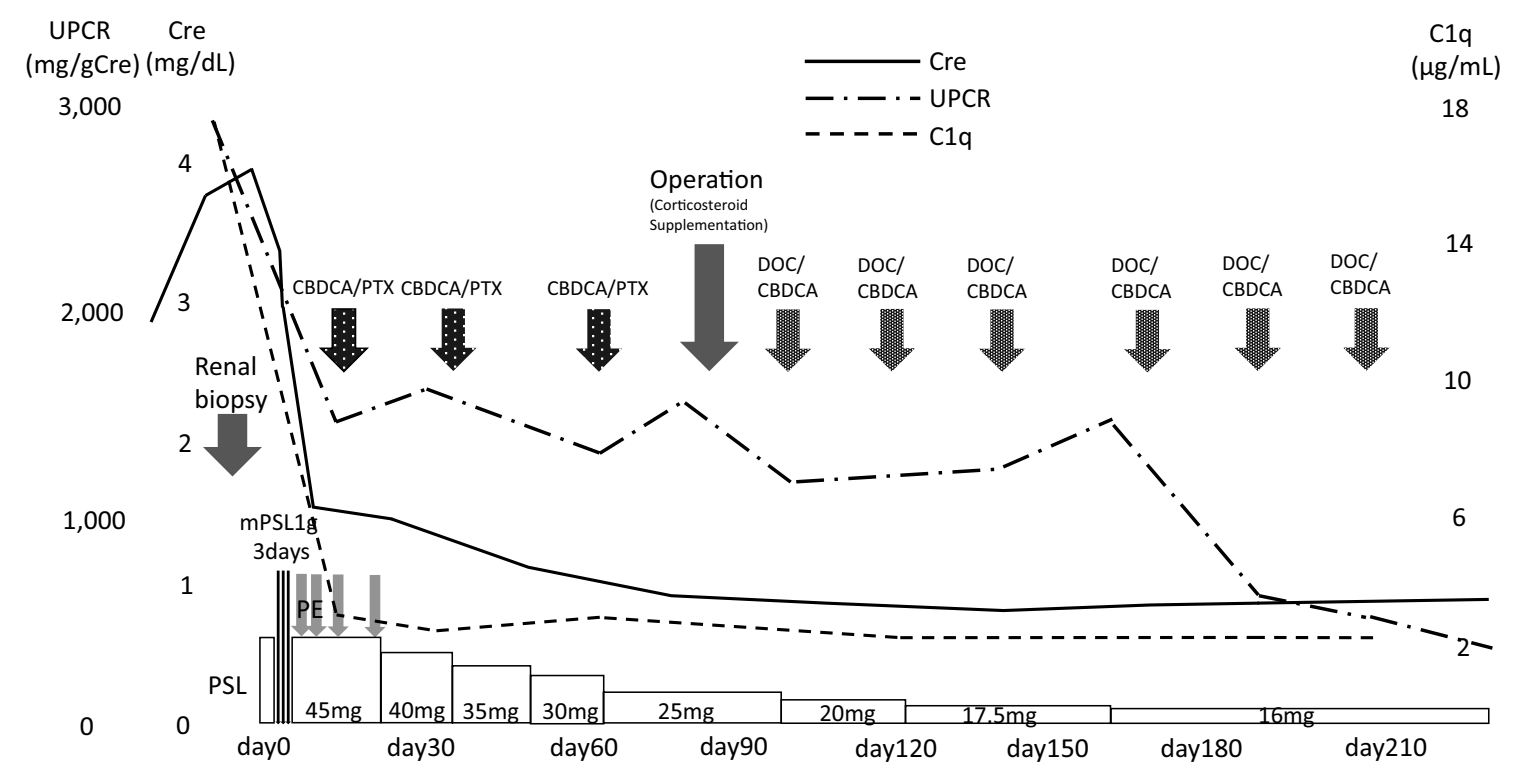

Figure 3. Clinical course of the patient. Oral prednisolone (1 mg/kg; $45 \mathrm{mg}$ ) was started, followed by intravenous methylprednisolone ( $1 \mathrm{~g} / \mathrm{day})$ for 3 days and plasma exchange. Chemotherapy with carboplatin and paclitaxel was also initiated, and prednisolone was tapered. The level of serum creatinine and immune complexes gradually decreased. She underwent abdominal total hysterectomy on day 90 and then received adjuvant chemotherapy with docetaxel and carboplatin. The serum levels of creatinine and immune complexes remained low.

(a)

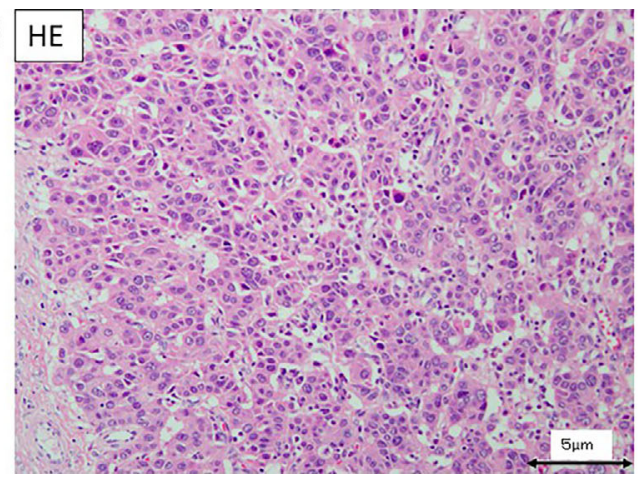

(b)

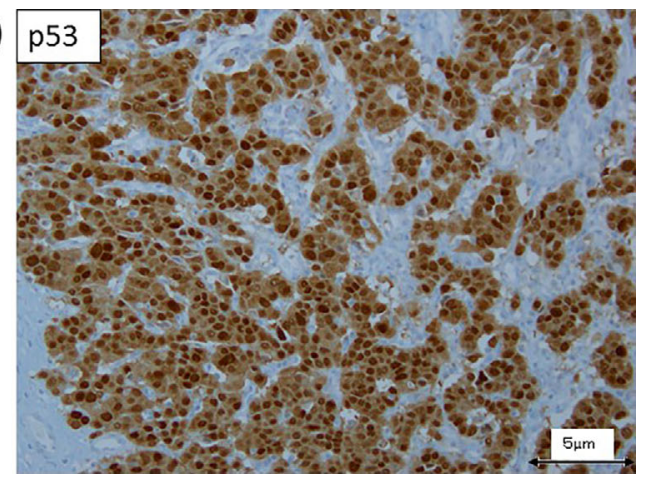

Figure 4. Histopathological findings for the ovarian tumor. (a) Nuclear pleomorphism. (b) Positive immunohistochemical staining for $\mathbf{p 5 3}$.

with SLE (malar rash, arthritis, serositis, positive antinuclear antibody, positive lupus erythematosus test) and ovarian dysgerminoma (14-16). No involvement of the hematologic, renal or central nervous systems was identified. The onset of SLE is thought to have been about one year prior to the diagnosis of ovarian cancer. Removal of the coexisting malignancy rapidly improved her arthritis and rash. Although the patient received melphalan six weeks after the surgery, she did not receive immunosuppressants or corticosteroid either before or after the surgery. She achieved lasting remission from SLE after 21 years. In the other case, a 54-year-old woman was diagnosed with SLE (lymphopenia, digital necrosis, positive antinuclear antibody, positive anti-dsDNA antibody, positive anticardiolipin antibody) and ovarian adenocarcinoma (17). No involvement of the renal or central nervous systems was seen. The onset of SLE is thought to have been one month prior to the diagnosis of ovarian cancer. Hydroxychloroquine and aspirin resulted in prompt clinical improvement. One month later, hysterectomy and omentectomy were performed, followed by treatment with carboplatin and paclitaxel. No information was provided on the clinical course after the surgery. The estimated SLEDAI score based on the articles was 10-11 for these 2 cases, which differed drastically from our case (SLEDAI score, 29). Neither of these previously reported patients received intensive immunosuppressants or corticosteroids either before or after the surgery, possibly due to their mild to moderate SLE activity.

Removal of the coexisting malignancy may play an important role in controlling SLE activity. However, our patient showed severe SLE activity including rapidly progressive glomerulonephritis, and a surgical approach was consid- 
ered a high risk. Corticosteroid therapy and plasma exchange were therefore started before chemotherapy and surgery. The oral ulcer and arthritis rapidly disappeared, and her renal function improved somewhat with corticosteroid therapy and plasma exchange, allowing for the initiation of chemotherapy. While the roles of corticosteroids and/or plasma exchange in SLE associated with ovarian cancer are yet to be confirmed, corticosteroids and/or plasma exchange prior to chemotherapy may be one option for improving the performance status in a patient.

Furthermore, the renal function in our patient further improved after surgery and chemotherapy with carboplatin and paclitaxel without immunosuppressive agents, suggesting that SLE might have been improved following the cancer treatment itself. Paclitaxel and docetaxel are known to be able to block the microtubule function and to inhibit the process of cell division, cell migration and cell adhesion. Indeed, paclitaxel has been shown to arrest mitosis and cell proliferation in synoviocytes (18). In addition, paclitaxel reduces the anti-dsDNA antibody titer in murine lupus (19). Carboplatin inhibits DNA synthesis in target cells, thus inhibiting tumor cell growth. Peripheral blood mononuclear cells from SLE patients have been shown to be sensitive to melphalan- or cisplatin-induced apoptosis $(20,21)$. The combination of corticosteroid, plasmapheresis and chemotherapy with carboplatin and paclitaxel may therefore be a valid treatment option for cases of SLE associated with ovarian cancer, although paclitaxel itself may cause autoimmune conditions, such as scleroderma-like skin changes, cutaneous lupus erythematosus and $\operatorname{SLE}(22,23)$.

All procedures performed in studies involving human participants were in accordance with the ethical standards of the institutional committee (Kitano Hospital Medical Research Institute Ethics Committee; approval number: S17-04-009) and with the 1964 Declaration of Helsinki and its later amendments.

Informed consent was obtained from the patient described in this case report.

The authors state that they have no Conflict of Interest (COI).

\section{References}

1. van Vollenhoven RF, Mosca M, Bertsias G, et al. Treat-to-target in systemic lupus erythematosus: recommendations from an international task force. Ann Rheum Dis 73: 958-967, 2014.

2. Cao L, Tong H, Xu G, et al. Systemic lupus erythematosus and malignancy risk: a meta-analysis. PloS One 10: e0122964, 2014.

3. Mao S, Shen H, Zhang J. Systemic lupus erythematosus and malignancies risk. J Cancer Res Clin Oncol 142: 253-262, 2016.

4. Yu KH, Kuo CF, Huang LH, Huang WK, See LC. Cancer risk in patients with inflammatory systemic autoimmune rheumatic diseases. A nationwide population-based dynamic cohort study in Taiwan. Medicine 95: e3540, 2016.
5. Chen YJ, Chang YT, Wang CB, Wu CY. Malignancy in systemic lupus erythematosus: A nationwide cohort study in Taiwan. Am J Med 123: 1150.e1-6, 2010.

6. Bernatsky S, Ramsey-Goldman R, Labrecque J, et al. Cancer risk in systemic lupus: an updated international multicenter cohort study. J Autoimmun 42: 130-135, 2013.

7. Bernatsky S, Ramsey-Goldman R, Foulkes WD, Gordon C, Clarke AE. Breast, ovarian, and endometrial malignancies in systemic lupus erythematosus: a meta-analysis. Br J Cancer 104: 1478-1481, 2011.

8. Goobie GC, Bernatsky S, Ramsey-Goldman R, Clarke AE. Malignancies in systemic lupus erythematosus: a 2015 update. Curr Opin Rheumatol 27: 454-460, 2015.

9. Petri M, Orbai AM, Alarcón GS, et al. Derivation and validation of the systemic lupus international collaborating clinics classification criteria for systemic lupus erythematosus. Arthritis Rheum 64: 2677-2686, 2012.

10. Tan EM, Cohen AS, Fries JF, et al. The 1982 revised criteria for the classification of systemic lupus erythematosus. Arthritis Rheum 25: 1271-1277, 1982.

11. Hochberg MC. Updating the American College of Rheumatology revised criteria for the classification of systemic lupus erythematosus. Arthritis Rheum 40: 1725, 1997.

12. Hansen JE, Chan G, Liu Y, et al. Targeting cancer with a lupus antibody. Sci Transl Med 4: 157ra142, 2012.

13. Noble PW, Chan G, Young MR, Weisbart RH, Hansen JE. Optimizing a lupus antibody for targeted cancer therapy. Cancer Res 75: 2285-2291, 2015.

14. Kahn MF, Ryckewaert A, Cannat A, Solnica J, de Seze S. Systemic lupus erythematosus and ovarian dysgerminoma: remission of the systemic lupus erythematosus after extirpation of the tumour. Clin Exp Immunol 1: 355-359, 1966.

15. Rotman M, Dorfmann H, de Sèze S, Kahn MF. Coexistence of systemic lupus erythematosus and of ovarian seminoma. Apparent rapid cure of the lupus after removal of the tumor. 7-year followup. Nouv Presse Med 1: 853-857, 1972.

16. Kahn MF, Solnica J, Bourgeois P. Disappearance of clinical and biological evidence of SLE after removal of an ovarian seminoma: 21 years' followup. J Rheumatol 13: 833-834, 1986.

17. Chtourou M, Aubin F, Savariault I, et al. Digital necrosis and lupus-like syndrome preceding ovarian carcinoma. Dermatology 196: 348-349, 1998.

18. Hui A, Kulkarni GV, Hunter WL, McCulloch CA, Cruz TF. Paclitaxel selectively induces mitotic arrest and apoptosis in proliferating bovine synoviocytes. Arthritis Rheum 40: 1073-1084, 1997.

19. Song YW, Kim HA, Baek HJ, Lee EB, Chung ES, Hong KM. Paclitaxel reduces anti-dsDNA antibody titer and BUN, prolonging survival in murine lupus. Int J Immunopharmacol 20: 669-677, 1998.

20. Souliotis VL, Vougas K, Gorgoulis VG, Sfikakis PP. Defective DNA repair and chromatin organization in patients with quiescent systemic lupus erythematosus. Arthritis Res Ther 18: 182, 2016.

21. Souliotis VL, Sfikakis PP. Increased DNA double-strand breaks and enhanced apoptosis in patients with lupus nephritis. Lupus 24: 804-815, 2015.

22. Dasanu CA, Alexandrescu DT. Systemic lupus erythematosus associated with paclitaxel use in the treatment of ovarian cancer. South Med J 101: 1161-1162, 2008

23. Adachi A, Horikawa T. Paclitaxel-induced cutaneous lupus erythematosus in patients with serum anti-SSA/Ro antibody. J Dermatol 34: 473-476, 2007.

The Internal Medicine is an Open Access journal distributed under the Creative Commons Attribution-NonCommercial-NoDerivatives 4.0 International License. To view the details of this license, please visit (https://creativecommons.org/licenses/ by-nc-nd/4.0/).

(C) 2019 The Japanese Society of Internal Medicine

Intern Med 58: 731-735, 2019 